\title{
LARGER MW-CLASS FLOATER DESIGNS WITHOUT UPSCALING? A DIRECT OPTIMIZATION APPROACH
}

\author{
Mareike Leimeister* \\ Naval Architecture, Ocean and Marine Engineering \\ University of Strathclyde \\ Glasgow G4 OLZ, United Kingdom \\ Division Load Calculation and System Dynamics \\ Fraunhofer IWES, Institute for Wind Energy Systems \\ Am Luneort 100, 27572 Bremerhaven, Germany
}

\author{
Athanasios Kolios, Maurizio Collu \\ Naval Architecture, Ocean and Marine Engineering \\ University of Strathclyde \\ Glasgow G4 OLZ, United Kingdom \\ Philipp Thomas \\ Division Load Calculation and System Dynamics \\ Fraunhofer IWES, Institute for Wind Energy Systems \\ Am Luneort 100, 27572 Bremerhaven, Germany
}

\section{ABSTRACT}

The trend towards larger offshore wind turbines (WTs) implies the need for bigger support structures. These are commonly derived from existing structures through upscaling and subsequent optimization. To reduce the number of design steps, this work proposes a direct optimization approach, by which means a support structure for a larger WT is obtained through an automated optimization procedure based on a smaller existing system. Due to the suitability of floating platforms for large $M W$ class WTs, this study is based on the OC3 spar-buoy designed for the NREL 5 MW WT. Using a Python-Modelica framework, developed at Fraunhofer IWES, the spar-buoy geometry is adjusted through iterative optimization steps to finally support a 7.5 MW WT. The optimization procedure focuses on the global system performance in a design-relevant load case. This study shows that larger support structures, appropriate to meet the objective of the hydrodynamic system behavior, can be obtained through automated optimization of existing designs without the intermediate step of upscaling.

Keywords: upscaling, automated design optimization, floating platforms, offshore wind turbines, Modelica, MoWiT, Python, IWT-7.5-164, OC3.

\footnotetext{
*Contact author: mareike.leimeister@iwes.fraunhofer.de.
}

$\begin{array}{ll}\text { NOMENCLATURE } \\ D_{\mathrm{BC}} & \text { Bottom cylinder diameter. } \\ D_{\mathrm{TC}} & \text { Top cylinder diameter. } \\ d_{\mathrm{BC}, \mathrm{b}} & \text { Distance to base of bottom cylinder. } \\ d_{\mathrm{BC}, \mathrm{t}} & \text { Distance to top of bottom cylinder. } \\ d_{\mathrm{TC}, \mathrm{b}} & \text { Distance to base of top cylinder. } \\ d_{\mathrm{TC}, \mathrm{t}} & \text { Distance to top of top cylinder. } \\ g & \text { Gravitational acceleration }\left(\approx 9.81 \mathrm{~m} / \mathrm{s}^{2}\right) . \\ h_{\mathrm{ballast}} & \text { Ballast filling height. } \\ h_{\mathrm{BC}} & \text { Height of bottom cylinder. } \\ K_{\mathrm{I}} & \text { Integral controller gain. } \\ K_{\mathrm{P}} & \text { Proportional controller gain. } \\ \zeta_{\mathrm{c}} & \text { Damping ratio of the response. } \\ \rho_{\mathrm{ballast}} & \text { Ballast density. } \\ \omega_{\mathrm{c}, \text { nat }} & \text { Controller natural frequency of the response. }\end{array}$

\section{INTRODUCTION}

Exploitation of deeper waters and installation of larger wind turbines (WTs) are current and future trends of the offshore wind industry. Different platforms are developed and prototypes of floating offshore wind turbines (FOWTs) are already installed and tested; however, floating support structures still need to 
conform with the increasing scale of WTs. The design process of (floating) wind turbine systems is very extensive and of iterative character. However, to avoid designing a floating support structure for a larger WT completely from scratch, advantage is taken of the experience with existing systems and upscaling procedures are used for dimensioning larger structures. Notwithstanding, subsequent optimization and modification are required to obtain an efficient final design, as standard upscaling procedures are only suitable for obtaining a first rough draft of an upscaled design. As discussed in [1], the theoretical scaling laws do not account for technological developments, such as lighter and high-strength materials for rotor blades, site-specific conditions and constraints, or further improvements due to, for example, economic interests. Thus, based on the upscaled support structure design, further modifications and optimization steps have to be performed until the final floater design for a larger WT is obtained.

To save effort, this work proposes the design of a floating platform for a larger WT, which is directly obtained through optimization, thus, eliminating the intermediate step of upscaling. By means of this approach, only a few initial adaptions are required to consider the changed WT weight, hence ensuring floatation, and to take account of the new WT tower base diameter. All other modifications of the floating support structure are covered within an optimization procedure, which is based on user-defined design variables, value ranges, and optimization criteria. The result of this automatic optimization is then a support structure design, which is suited for a pre-defined larger WT and specified site, and is also optimized with respect to user-defined criteria.

In this paper, the OC3 (Offshore Code Comparison Collaboration) phase IV spar-buoy FOWT system [2] serves as basis for the application of the direct optimization approach. By this means, a support structure for a 7.5 MW WT (Fraunhofer's IWT7.5-164 [3]) shall be obtained from a floater, which is initially designed for a 5 MW WT (NREL 5 MW [4]). Thus, first, the reference systems are introduced. Afterwards the methodology is explained, comprising the model setup of the FOWT system and the generic structure and functionality of the optimization framework. On this basis, the specific design conditions, such as the design variables, optimization criteria, simulation load case, and optimization parameters, are set. The results show the development of the design throughout the optimization process, as well as the final floater design for the 7.5 MW WT and its performance. Some discussion and conclusion round off this paper.

\section{REFERENCE CASE}

In this study, the OC3 spar-buoy [2], supporting the NREL $5 \mathrm{MW}$ WT [4], is used as basis FOWT system and hence as input

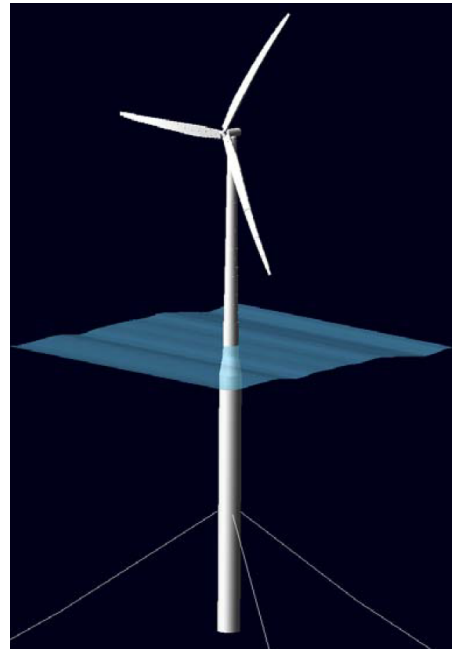

FIGURE 1. THE OC3 SPARBUOY FOWT SYSTEM [2].

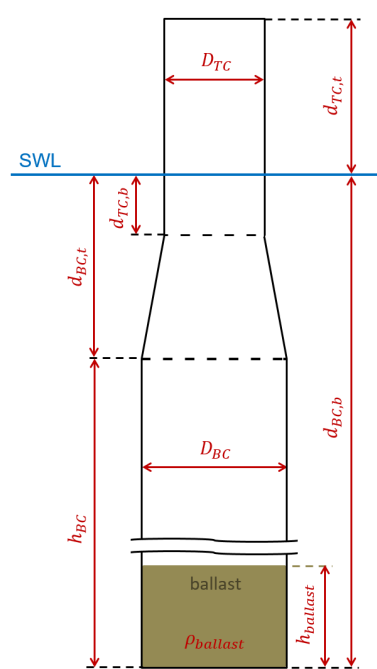

FIGURE 2. PARAMETERS OF THE OC3 SPAR-BUOY. to the direct optimization approach, with the final goal to obtain a floating spar-buoy, which suits the 7.5 MW IWT-7.5-164 WT [3]. These two reference structures are introduced hereinafter.

\subsection{The Basis FOWT System}

The OC3 phase IV FOWT system [2] consists of a floating spar-buoy, which supports the NREL 5 MW WT [4], as visualized in Fig. 1. The main parameters of the FOWT system are given in Tab. 1, focusing on the spar-buoy geometry, as this is to be modified during the direct optimization approach, but also including the system masses, to be able to correct for the changed masses of tower and rotor-nacelle assembly (RNA) of the WT on top of the floater when scaling up. The geometrical parameters are defined according to Fig. 2, with the distances set in relation to the still water level (SWL).

\subsection{The Larger MW-Class WT}

The target WT size to be supported by the optimized floating spar-buoy, is the IWT-7.5-164 [3], a 7.5 MW reference WT designed by Fraunhofer IWES (Institute for Wind Energy Systems). For this WT, different support structures (including the tower) exist for an onshore system or two different offshore systems. The monopile-based offshore WT system (TANDEM design [3]) is used in this study, however, the monopile is removed and the tower is cut at $10.0 \mathrm{~m}$ above SWL, which corresponds to the top of the floating platform. This way, the hub height of the IWT-7.5-164 remains unchanged. The main WT parameters, considering the shortened tower, are presented in Tab. 2. 
TABLE 1. PROPERTIES OF THE OC3 FOWT SYSTEM $[2,4]$.

\begin{tabular}{lcr}
\hline Parameter & Symbol & \multicolumn{1}{c}{ Value } \\
\hline RNA mass & & $350.0 \mathrm{t}$ \\
Tower mass & & $249.7 \mathrm{t}$ \\
Hub height & & $90.0 \mathrm{~m}$ \\
Tower base diameter & & $6.5 \mathrm{~m}$ \\
Top cylinder diameter & $D_{\mathrm{TC}}$ & $6.5 \mathrm{~m}$ \\
Bottom cylinder diameter & $D_{\mathrm{BC}}$ & $9.4 \mathrm{~m}$ \\
Distance to top of top cylinder & $d_{\mathrm{TC}, \mathrm{t}}$ & $10.0 \mathrm{~m}$ \\
Distance to base of top cylinder & $d_{\mathrm{TC}, \mathrm{b}}$ & $4.0 \mathrm{~m}$ \\
Distance to top of bottom cylinder & $d_{\mathrm{BC}, \mathrm{t}}$ & $12.0 \mathrm{~m}$ \\
Distance to base of bottom cylinder & $d_{\mathrm{BC}, \mathrm{b}}$ & $120.0 \mathrm{~m}$ \\
Height of bottom cylinder & $h_{\mathrm{BC}}$ & $108.0 \mathrm{~m}$ \\
Platform mass including ballast & & $7,466.3 \mathrm{t}$ \\
\hline
\end{tabular}

TABLE 2. PROPERTIES OF THE IWT-7.5-164 [3].

\begin{tabular}{lc}
\hline Parameter & Value \\
\hline RNA mass & $536.8 \mathrm{t}$ \\
Tower mass from 10 m above SWL & $491.5 \mathrm{t}$ \\
Hub height & $111.6 \mathrm{~m}$ \\
Tower top diameter & $3.0 \mathrm{~m}$ \\
Tower base diameter & $7.0 \mathrm{~m}$ \\
\hline
\end{tabular}

\section{METHODOLOGY}

To generate a new floater design for a larger MW-class WT on the basis of a smaller existing FOWT system, first, the FOWT system has to be modelled, comprising the basis design, replacing the WT, and adjusting some parameters to ensure system conformity. This model is then further processed within an optimization framework. The modeling and optimization environments are described in the following.

\subsection{Model Setup}

The FOWT system is modeled in MoWiT (Modelica for Wind Turbines) ${ }^{1}$, a library established at Fraunhofer IWES.

\footnotetext{
${ }^{1}$ Formerly OneWind Modelica library.
}

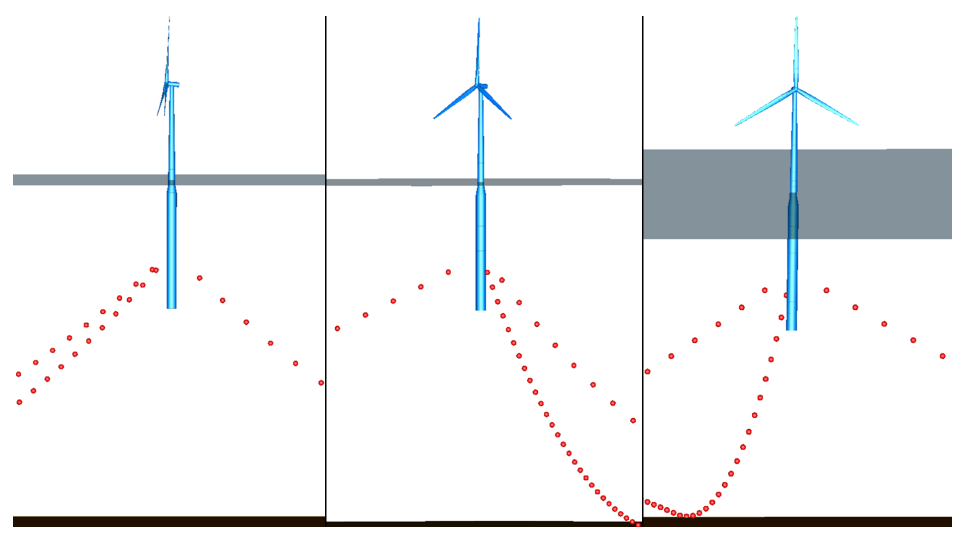

FIGURE 3. THE OC3 FOWT SYSTEM MODELLED IN MoWiT AND SIMULATED IN DYMOLA [5].

MoWiT uses the object-oriented and equation-based modeling language Modelica and is capable of performing fully-coupled aero-hydro-servo-elastic simulations of WT systems. The hierarchical structure and multibody approach in Modelica allow for component-based modelling, which is of high value for the purpose of this study. With respect to the hydrodynamics, which are of high importance for a floating system, the MoWiT library accounts for hydrodynamic loads following a modified version of the MacCamy-Fuchs approach (to consider the relative motion between the structure and the fluid in addition to capturing the variation of the added inertia coefficient and including diffraction effects), as well as the drag term of the Morison equation (for taking into account the viscous forces). The buoyancy force and righting moment are calculated in time-domain depending on the actual local surface elevation and the current position of the FOWT system. [5-7]

\subsubsection{Implementation of the OC3 FOWT in MoWiT} According to the definitions given in [2], the OC3 FOWT system is implemented in MoWiT as visualized in Fig. 3, using Dymola as simulation engine. The modeling happens component-based as explained in [5], meaning that the entire system is split up into separate models for the support structure - comprising tower, floater, and mooring lines -, the nacelle with drivetrain and generator, the rotor - covering the blades and the hub -, the operating control, as well as two environmental models for wind and waves, including currents. In a separate study [8] the model of the spar-buoy FOWT is verified, using the code-to-code comparisons within the OC3 phase IV project [9].

2.1.2 Initial Adaptions Due to the component-based MoWiT library, the original NREL 5 MW WT can easily be replaced by the IWT-7.5-164 WT by exchanging the models for rotor, nacelle, and operating control, as well as the tower 
submodel within the support structure model. However, due to the different WT dimensions (mass and tower base diameter), some initial adaptions are required before the model can be given as input to the optimization framework.

Thus, the spar-buoy geometry is partially modified by increasing the top cylinder diameter from the initial $6.5 \mathrm{~m}$ to $7.0 \mathrm{~m}$ to fit the new tower base diameter of the IWT-7.5-164. This changed parameter affects the floater structural mass for the top cylinder and the tapered part up to $12.0 \mathrm{~m}$ below SWL, but also the displaced water volume from $12.0 \mathrm{~m}$ below SWL to the water line. The change in the equivalent buoyancy mass and the structural mass can be determined directly from the original and modified spar-buoy geometry. Hence, the buoyancy mass is increased by around $46.7 \mathrm{t}$, while the floating structure becomes heavier by $8.9 \mathrm{t}$. Furthermore, the exchanged WT on top of the floater results in an additional mass increase by $428.6 \mathrm{t}$. These changed buoyancy and structural masses are accounted for in the determination of the ballast height, which is internally calculated in the model to ensure floatation of the FOWT system, and yield a ballast filling height of $45.38 \mathrm{~m}$ for this initially adapted FOWT system.

Furthermore, the WT controller needs to be adjusted to avoid negative aerodynamic damping, as now the IWT-7.5-164 is on top of a floating platform. For this purpose, the integral and proportional controller gains $K_{\mathrm{I}}$ and $K_{\mathrm{P}}$ are modified, following the general descriptions and recommendations given in [10], as well as adopting the approach used in [1]. Hence, the damping ratio of the response $\zeta_{c}$, required for the determination of $K_{\mathrm{P}}$, is set equal to 0.7. In addition, the controller natural frequency of the response $\omega_{\mathrm{c}, \text { nat }}$ needs to be defined. As the controller should be slower than the system response, the pitch natural frequency of the OC3 FOWT system, obtained by the MoWiT model, is taken and reduced by a factor of 1.3 , according to [1]. This yields a controller natural frequency of $\omega_{\mathrm{c}, \text { nat }}=0.1527 \mathrm{rad} / \mathrm{s}$. Together with the 7.5 MW WT specific drivetrain and blade-pitch controller parameters, the controller gains are computed and set to $K_{\mathrm{I}}=0.00141924$ and $K_{\mathrm{P}}=0.01300953 s$.

\subsection{Optimization Framework in Python}

This model of the FOWT system, consisting of the OC3 spar-buoy and the IWT-7.5-164 WT with the required initial adaptions, as described in Section 2.1, is given as input to the optimization framework. The optimization framework itself is based on the Python-Modelica framework, developed at Fraunhofer IWES for automated simulation, using the extension for automated optimization [11]. Working with Python as programming interface, existing packages can be utilized, by which means the interface between the programming tool Python, the modeling environment Modelica, and the simulation engine Dymola is already defined. In the basic Python-Modelica framework [11], the MoWiT model is processed, parameters are modified and defined, simulations are managed for parallel or consecutive execution, and scripts for writing output files are set up. This basic Python-Modelica framework is extended by incorporation of optimization functionalities, so that optimization tasks can be performed with the provided MoWiT model.

The optimization functionalities comprise the definition of the optimization problem, the optimizer, and the optimization algorithm. To define the optimization problem, design variables of the MoWiT FOWT system model have to be specified, which are customizable during the optimization loops. The values or ranges of values, which these design variables are allowed to take on, are as well prescribed within the optimization problem definition. Furthermore, the objectives of the optimization process and - if existing - corresponding constraints have to be specified. For the optimizer, there are several available in open-source frameworks for the implementation in Python. There is as well a huge variety of optimization methods and their capabilities. Hence, optimizers could be gradient-based or gradient-free, could be based for instance on the Newton approach, quadratic programming, evolutionary algorithms, or the particle swarm theory, and some might be capable to process multi-objective optimization tasks [12]. Finally, the optimization algorithm follows the method of the used optimizer to select in each iteration new design variables from the prescribed value ranges and base this choice on the performance - with respect to objectives and constraints - of the previously simulated system designs. The algorithm is executed until a specified stop criterion, which might be a maximum number of iterations or a convergence tolerance, is reached. [11,12]

For more detailed information on the Python-Modelica framework for automated simulation and optimization, as well as on the optimization functionalities themselves, the reader is referred to [11] and [12], respectively.

\section{DESIGN CONDITIONS}

The specific settings for the application of the optimization framework, described in Section 2.2, to the adjusted FOWT system model, presented in Section 2.1, are outlined hereinafter.

\subsection{Design Variables}

The object of interest in this study is the floating spar-buoy structure; however, the upper cylindrical part is already adjusted in the initial model set up to fit the larger tower base diameter of the 7.5 MW WT. Hence, the design variables, which are to be modified during the direct optimization approach, concern the bottom cylinder geometry $\left(D_{\mathrm{BC}}\right.$ and $\left.h_{\mathrm{BC}}\right)$, as well as the ballast. 
TABLE 3. DESIGN VARIABLES, THEIR VALUE RANGES, AND THEIR VALUES IN THE INITIAL FOWT SYSTEM MODEL.

\begin{tabular}{lccc}
\hline Variable & Lower bound & Upper bound & Initial value \\
\hline$D_{\mathrm{BC}}$ & $7.0 \mathrm{~m}$ & $10.0 \mathrm{~m}$ & $9.4 \mathrm{~m}$ \\
$h_{\mathrm{BC}}$ & $68.0 \mathrm{~m}$ & $108.0 \mathrm{~m}$ & $108.0 \mathrm{~m}$ \\
$\rho_{\text {ballast }}$ & $1,281.0 \mathrm{~kg} / \mathrm{m}^{3}$ & $2,600.0 \mathrm{~kg} / \mathrm{m}^{3}$ & $1,907.1 \mathrm{~kg} / \mathrm{m}^{3}$ \\
\hline
\end{tabular}

The latter is defined through its density $\rho_{\text {ballast }}$ and filling height $h_{\text {ballast }}$ within the bottom cylinder. As the ballast density is prescribed by available filling materials, it is selected as the third design variable, while the ballast height is not a direct design variable as it depends on the system parameters and chosen design variables and is determined internally to ensure floatation of the WT system. With regard to the structural parameters, only the diameter and height of the bottom cylinder are selected as design variables, whereas the original wall thickness remains unchanged, as the objective of the optimization is not the design strength but rather focuses on the hydrodynamic behavior of the FOWT system. Another component belonging to the floating spar-buoy structure is the mooring system. This, however, implying also the attachment points (fairleads), remains unchanged as the yaw stability is not analyzed explicitly in this study, but it would have to be considered as design variable as well, if the entire system stability is to be optimized.

For the three design variables, the allowable value ranges, as well as their values in the initial adjusted FOWT design are presented in Tab. 3. For the spar-buoy bottom cylinder diameter the lower limit is prescribed by the tower base diameter of the IWT-7.5-164, while the upper limit is inspired by the original value and chosen to be not significantly larger for the ease of handling and manufacturing. For the same reasons, the original bottom cylinder height is set as upper limit for this design variable, with an option to be reduced by up to $40.0 \mathrm{~m}$. The potential ballast density values originate from the density ranges of cheap materials, such as sand with varying water content, rocks, or clay $[13,14]$. Apart from the ballast type, which is conditional on available lower-cost material, the upper bounds for the geometric parameters are shaped by the author's additional objective of limiting the outer dimensions of the floating system to facilitate the handling and manufacturing processes, but also to prevent a significant loss in the structural strength as this was excluded from the analysis. Different user-specific objectives and set value ranges would definitely affect the results. This is addressed again in the discussion at the end of the paper.
TABLE 4. OBJECTIVES OF THE OPTIMIZATION, THEIR TARGET VALUES, AND THEIR CONSTRAINTS.

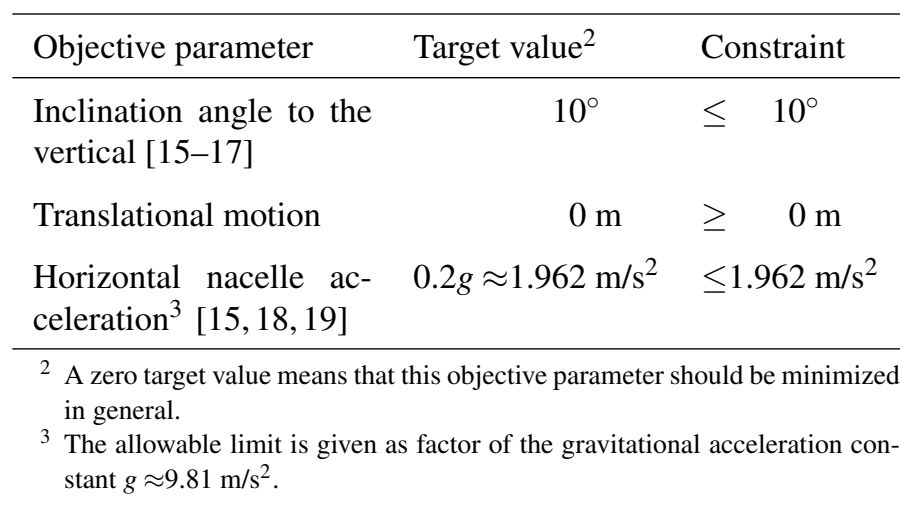

Within the formulation for determining the ballast filling height as a function of the system mass and buoyancy mass (both are directly derived from the geometric parameters), it is checked that $h_{\text {ballast }}$ never becomes negative, nor exceeds $h_{\mathrm{BC}}$. If the settings of the design variables would violate one of these constraints, either the ballast density or floater material density is modified to overcome this issue. With having this defined within the MoWiT model, it is ensured that the ballast height remains within these boundaries during the entire optimization iterations.

\subsection{Optimization Objectives}

The objective of applying the direct optimization approach to the adapted OC3 spar-buoy carrying the IWT-7.5-164 WT is to obtain an appropriate floating support structure for the 7.5 MW WT, so that this FOWT system is stable and complies with general global performance criteria. This study neither focuses on the structural strength nor on the loading on the structure, such as fatigue due to system eigenfrequencies. These aspects, however, can be integrated by adding more optimization objectives, which are based on post-processed parameters and analyses.

Thus, focusing for the time being only on the global performance of the FOWT in operation, three objective functions are defined. These comprise the platform total inclination angle for stability and WT operational reasons, the translational motions due to motion restrictions of the power cable, and the nacelle acceleration because of sensitive components within the nacelle.

To avoid overdimensioning of the FOWT system, which would only yield dispensable high safety factors but an overpriced design, common operational limits are set as objectives. The target values and constraints are summarized in Tab. 4. As, 
however, the optimization objectives have to be provided in terms of objective functions which are to be minimized, the three objectives follow the expressions given in Eqn. 1 to Eqn. 3, respectively, normalizing the objectives for inclination (Eqn. 1) and acceleration (Eqn. 3) with respect to their target values.

$$
\frac{\mid \text { inclination }-10^{\circ} \mid}{10^{\circ}}
$$

translation

$$
\frac{\mid \text { acceleration }-1.962 \frac{\mathrm{m}}{\mathrm{s}^{2}} \mid}{1.962 \frac{\mathrm{m}}{\mathrm{s}^{2}}}
$$

These objective functions are evaluated during the optimization for each considered design solution, substituting the objectives (inclination, translation, and acceleration) in each case by the maximum value obtained during the simulation. This is directly derived by evaluating the resulting time series.

\subsection{Design-Relevant Load Case}

The iterative optimization procedure is carried out based on design-relevant load cases, used to simulate the considered floating system and to derive the performance criteria from the simulation results. For this first utilization of the direct optimization approach, only one design-relevant load case is selected based on DLC (design load case) simulations with the original OC3 sparbuoy FOWT system. From the recommended DLCs in the international standard IEC 61400-3 [20], the following DLCs are selected for the simulation with the OC3 floating system, with having the optimization objectives, defined in Section 3.2, in mind:

- DLC 1.1 around rated wind speed (power production, normal turbulent wind model, normal sea state):

In normal power production around rated wind speed, the highest thrust force, and hence, a large inclination angle and large horizontal displacement are expected.

- DLC 1.3 below, at, and above rated wind speed (power production, extreme turbulent wind model, normal sea state):

If the WT is sensitive to wind, hence at a wind dominated site, the extreme turbulent wind may yield extreme fluctuations and therefore large values for the nacelle acceleration.

- DLC 1.6a below, at, and above rated wind speed (power production, normal turbulent wind model, severe sea state): If the WT is sensitive to waves, hence at a wave dominated site, the severe sea state may yield extreme fluctuations and therefore large values for the nacelle acceleration.

- DLC 6.1b (parked condition, extreme steady wind model, reduced wave height model, both with 50-year recurrence period):

In such an extreme event with 50-year recurrence period, the highest loads, implying a large inclination angle and a large horizontal displacement, are expected.

When running the DLC simulations, the OC3 FOWT system showed to be not designed properly for such an extreme environmental condition given in DLC 6.1b. As this is the only DLC from the four selected ones, in which the WT is not operating, there is no need to consider separate (less restrictive) objectives for a FOWT system in parked condition. Thus, for the evaluation, only the other three DLCs $(1.1,1.3,1.6 \mathrm{a})$ were used and it turned out that DLC 1.6a at rated wind speed and with a yaw misalignment angle of $8^{\circ}$ yields the highest values in the three objective parameters defined in Section 3.2. This environmental condition is transferred to the IWT-7.5-164 FOWT system, assuming similar system behavior, and used for the simulations within the direct optimization approach.

\subsection{Optimization Settings}

From a broad range of algorithms and optimization procedures, of which some are presented in [11], the evolutionary algorithm NSGAII (non-dominated sorting genetic algorithm II) from Platypus [21] is selected to be used in this study. The reasons for this choice are, first of all, the demands from the system model and optimization task under consideration. Due to the complexity of a FOWT system, gradient-based optimizers would not be suitable as the system equations cannot be reduced to just one single equation. Furthermore, the three explicitly defined objective functions, outlined in Section 3.2, recommend the utilization of a multi-objective optimizer. Secondly, taking into account these demands, three common state-of-the-art gradient-free multi-objective optimizers [22] NSGAII, NSGAIII (non-dominated sorting genetic algorithm III), and SPEA2 (strength Pareto evolutionary algorithm) are tested, using the open-source framework Platypus [21]. The comparison showed that NSGAII more accurately meets the constraints and objectives, better than NSGAIII, but is at the same time, compared to SPEA2, faster converging to an optimum solution.

For these reasons, the direct optimization approach is carried out with the NSGAII optimizer from Platypus. As this method belongs to the group of evolutionary algorithms, which basically follow the principle of Darwin's theory of evolution, the optimizer requires figures for the population size and number of generations as input [11]. For this first direct optimization application, the population size is set to 36 individuals in each gener- 
ation and the total number of simulations to be run is defined as 1080 , which would correspond to 30 generations (29 plus the start population with number 0$)^{4}$. A comprehensive sensitivity analysis for justifying the selected population size and number of generations was not yet covered in this study. However, based on the convergence behavior, discussed in Section 4.1, the chosen settings proved to provide satisfactory results.

\section{RESULTS}

The results of the direct optimization approach, applied to the initially adapted OC3 spar-buoy with the IWT-7.5-164 on top, are presented in the following, first, showing the results throughout the optimization process, and then focusing on the final floater design, which is obtained from the direct optimization approach to be proper for supporting the IWT-7.5-164.

\subsection{Development Throughout the Optimization Itera- tions}

Within the direct optimization procedure, finally, 1097 individuals are created, simulated, and evaluated. The number differs slightly from the specified number of total simulations of 1080, as defined in Section 3.4, due to the internal management of running simulations in parallel. This way, individuals from generation 0 up to generation 31 are created, while the last full generation, meaning containing all 36 individuals, is generation number $26^{4}$.

Within each generation, the design variables for the individuals are chosen by the optimizer from the specified value ranges, based on the objectives obtained from the previously simulated individuals. Hence, a trend from a broad spread of selected values for the design variables towards more and more optimum values is clearly visible throughout the optimization procedure, as visualized in Fig. 4(a). The corresponding trend in the obtained optimization objectives (Fig. 4(b)) shows as well decreasing spreads and, what is most important, an improvement with respect to the objectives set. In Fig. 4(b), it is noticeable that in the first few generations not for all 36 individuals an objective value is plotted. The reason for this is that, at the start of the optimization procedure, individuals from the entire allowable value ranges for the design variables are created. These, however, do not all perform that well and some might be instable, which causes an early termination of the simulation. The effectively simulated time is taken as criterion for identifying unsuccessful simulations, which are then assigned undesirable objectives to ensure exclusion of these designs $[11,12]$.

\footnotetext{
${ }^{4}$ Note: The counting of generations starts with number 0 for the start population.
}

\subsection{Spar-Buoy Design for the IWT-7.5-164}

From the 1097 individuals, obtained within the direct optimization approach, the optimum and final appropriate spar-buoy floater design for the IWT-7.5-164 WT has to be found. The method and criteria for the selection of the optimum geometry are described firstly, and then the obtained FOWT system is presented and analyzed.

4.2.1 Selection of the Floater Design As the specified stop criterion, defined through the total number of simulations, which corresponds approximately to a maximum number of generation, is set quite arbitrary, first, the generation has to be determined at which the optimization algorithm has converged before it starts diverging again. To do so, two selection options are considered: based on the minimum spread of the design variables or based on the minimum spread of the objectives. The first option would yield generation 23, the latter generation 22 to be considered for determining the optimum individual, as highlighted in Fig. 4(a) and Fig. 4(b) in dark red, respectively. As the point of interest in this study is the convergence of the optimization to one optimum design, the first selection method, based on the minimum spread of the design variables, is used, and hence generation 23 is taken for finding the best individual in it.

For the selection of the optimum individual, first, the minimum value for each of the three objectives is identified, comparing all individuals within generation 23. These three values, which originate from different individuals in generation 23, are used as utopia for the objectives. Then, for each individual in generation 23, the distance of its objectives to the utopia point is determined by means of the root of the sum of the three individual distances squared. Here, it has to be noted that the distances for the inclination and acceleration objectives are calculated directly, as these objectives are already normalized, as indicated in Eqn. 1 and Eqn. 3, respectively, while the distance for the translation objective, which is by definition (see Eqn. 2) just directly the translation value, is now normalized with respect to the utopia value for the translation. Doing so, the individual within generation 23, which yields the minimum distance of its objectives to the utopia point, is determined to be the optimum solution.

4.2.2 The Final Floater Geometry Following the selection approach, described in Subsection 4.2.1, the final floater geometry of the most appropriate spar-buoy design to support the IWT-7.5-164 is obtained. Its values for the design variables are presented in Tab. 5. This also demonstrates that all values lie within the specified allowable value ranges. A schematic drawing, comparing the original OC3 spar-buoy for the NREL $5 \mathrm{MW}$ WT, with the initially adapted OC3 floater for the IWT-7.5-164 WT, which dealt as input to the 


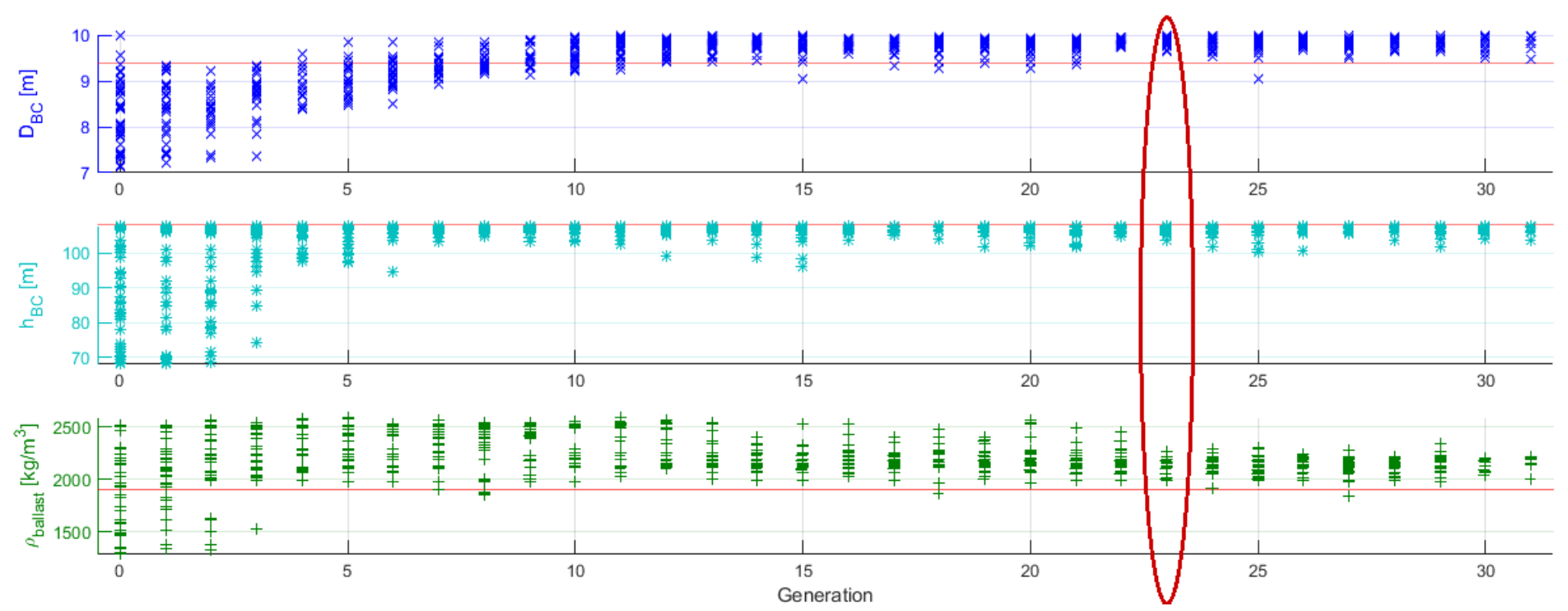

(a) DEVELOPMENT OF THE DESIGN VARIABLES IN COMPARISON TO THE INITIAL VALUES (RED LINES).
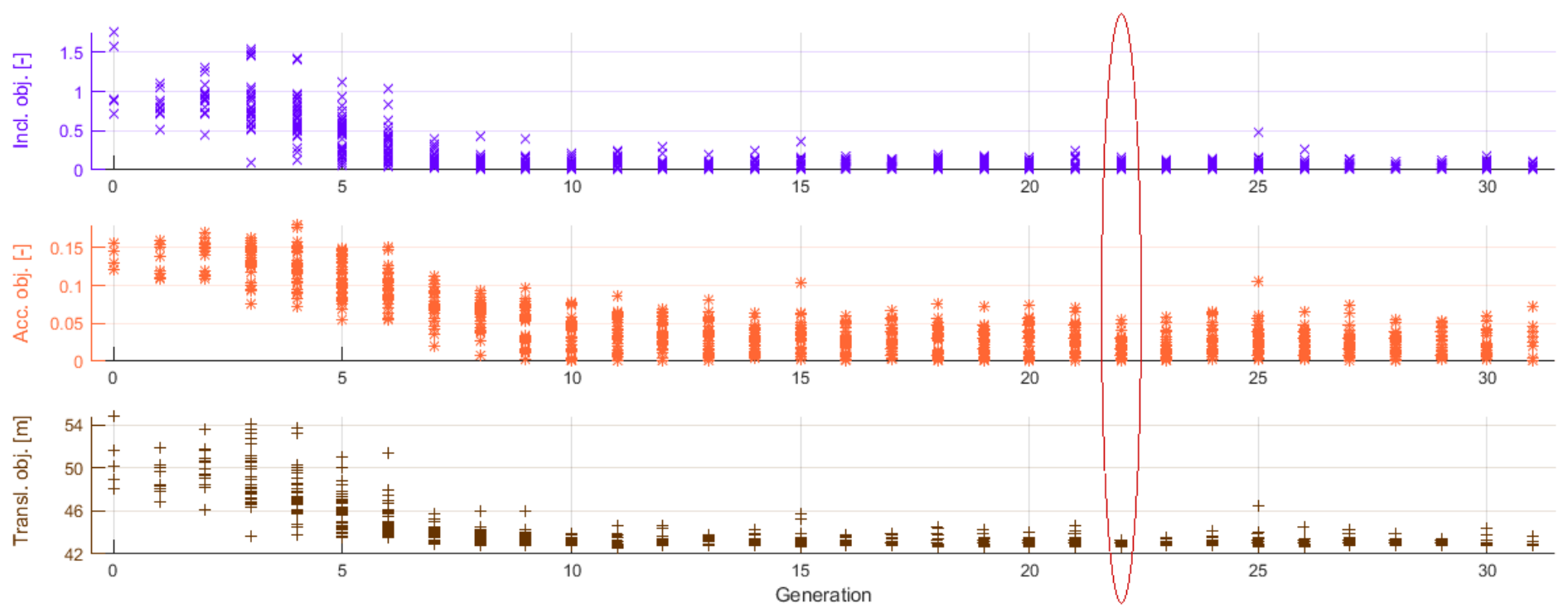

(b) DEVELOPMENT OF THE OPTIMIZATION OBJECTIVES.

FIGURE 4. SELECTED INDIVIDUALS THROUGHOUT THE SIMULATED GENERATIONS IN THE DIRECT OPTIMIZATION APPROACH, THE GENERATION SELECTED BASED ON THE FIRST AND USED APPROACH (THICK LINE) OR THE SECOND SELECTION OPTION (THIN LINE) IS CIRCLED IN DARK RED.

direct optimization approach, and the final optimized spar-buoy geometry for the IWT-7.5-164 WT, is shown in Fig. 5.

Due to the fact that two potential methods have been initially considered for the selection of the generation, at which the optimization has converged, as mentioned in Subsection 4.2.1, the results obtained when using the second alternative based on the minimum spread of the objectives (yielding generation 22) are determined as well and compared in Tab. 6 to the final results (from the selected generation 23) presented above. This shows that, for both obtained optimum individuals, the spar-buoy design variables, as well as the calculated distance of the objectives to the utopia point differ only marginally. But still, the final optimum spar-buoy geometry, selected from the individuals within generation 23, and hence following the approach as chosen and described in Subsection 4.2.1, scores higher. 
TABLE 5. FINAL VALUES OF THE DESIGN VARIABLES AND THEIR SPECIFIED VALUE RANGES.

\begin{tabular}{lccc}
\hline Variable & Final value & \multicolumn{2}{c}{ Allowable value range } \\
\hline$D_{\mathrm{BC}}$ & $9.89 \mathrm{~m}$ & $7.0 \mathrm{~m}-10.0 \mathrm{~m}$ \\
$h_{\mathrm{BC}}$ & $106.42 \mathrm{~m}$ & $68.0 \mathrm{~m}-108.0 \mathrm{~m}$ \\
$\rho_{\text {ballast }}$ & $2,126.5 \mathrm{~kg} / \mathrm{m}^{3}$ & $1,281.0 \mathrm{~kg} / \mathrm{m}^{3}-2,600.0 \mathrm{~kg} / \mathrm{m}^{3}$ \\
\hline
\end{tabular}

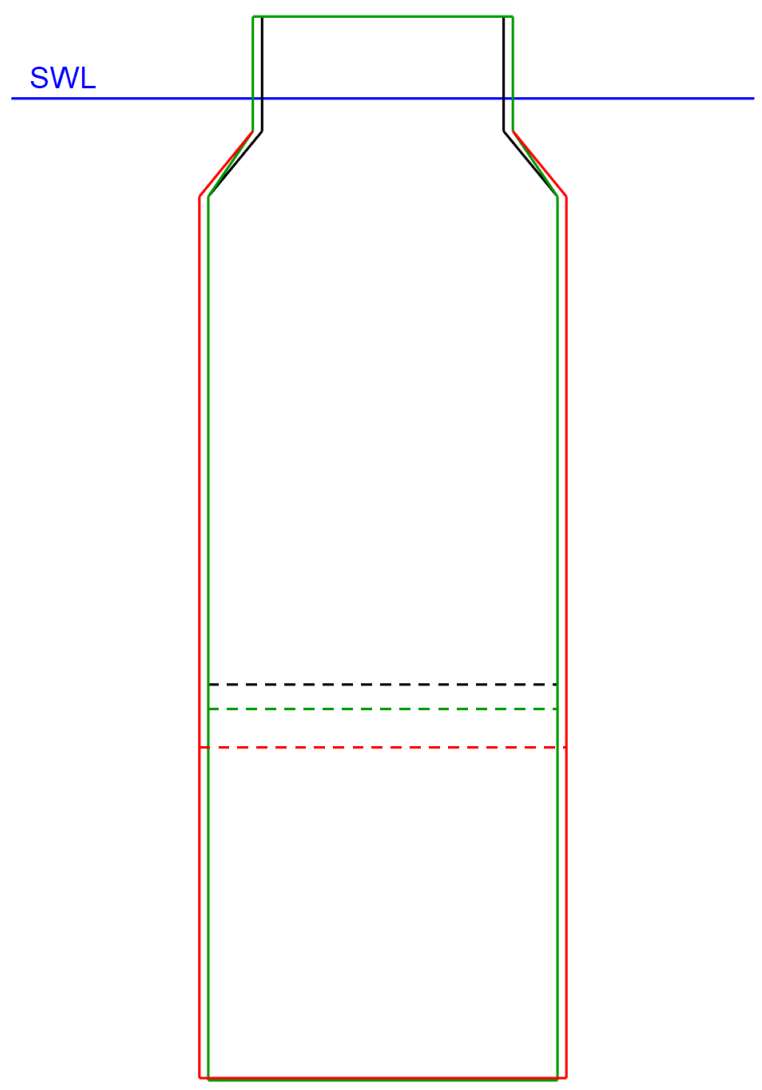

- original OC3 spar-buoy for the NREL 5MW

— initially adapted OC3 spar-buoy for the IWT-7.5-164

— optimized spar-buoy floater for the IWT-7.5-164

FIGURE 5. ORIGINAL, INITIALLY ADAPTED, AND FINAL OPTIMUM SPAR-BUOY FLOATER GEOMETRIES IN COMPARISON, DASHED LINES INDICATING THE BALLAST HEIGHT.

\subsubsection{Performance of the 7.5 MW FOWT System} To prove the suitability of the obtained final spar-buoy geometry, the performance of the FOWT system, consisting of the IWT7.5-164 WT and the optimum floater design, outlined in Subsection 4.2.2, is analyzed. This means that the considered design-
TABLE 6. COMPARISON OF THE RESULTS FOR THE OPTIMUM SPAR-BUOY FLOATER GEOMETRY FOR THE TWO GENERATION SELECTION METHODS.

\begin{tabular}{lcc}
\hline Variable & Generation 23 & Generation 22 \\
\hline$D_{\mathrm{BC}}$ & $9.89 \mathrm{~m}$ & $9.88 \mathrm{~m}$ \\
$h_{\mathrm{BC}}$ & $106.42 \mathrm{~m}$ & $106.42 \mathrm{~m}$ \\
$\rho_{\text {ballast }}$ & $2,126.5 \mathrm{~kg} / \mathrm{m}^{3}$ & $2,114.4 \mathrm{~kg} / \mathrm{m}^{3}$ \\
Distance to utopia point & 0.0218 & 0.0254 \\
\hline
\end{tabular}

TABLE 7. PERFORMANCE OF THE FINAL DESIGN WITH RESPECT TO THE DEFINED OPTIMIZATION OBJECTIVES.

\begin{tabular}{lccl}
\hline $\begin{array}{l}\text { Objective param- } \\
\text { eter }\end{array}$ & Maximum & Target & Constraint \\
\hline Inclination & $9.86^{\circ}$ & $10^{\circ}$ & $\leq 10^{\circ}$ \\
Translation & $42.92 \mathrm{~m}$ & $0 \mathrm{~m}$ & $\geq 0 \mathrm{~m}$ \\
Acceleration & $1.929 \mathrm{~m} / \mathrm{s}^{2}$ & $1.962 \mathrm{~m} / \mathrm{s}^{2}$ & $\leq 1.962 \mathrm{~m} / \mathrm{s}^{2}$ \\
\hline
\end{tabular}

relevant load case, according to the definition in Section 3.3, is simulated with the obtained 7.5 MW FOWT system and the maximum value for each of the defined optimization objective, specified in Section 3.2, is taken from the output time series. The results are presented in Tab. 7 and it can clearly be seen that all constraints are met, while still the objective parameters are close to the target values.

\section{DISCUSSION}

By means of the presented direct optimization approach, a floating support structure design for a larger WT is obtained based on a smaller existing FOWT system. The finally achieved design is based on the specified design conditions, as described in Sections 3.1 to 3.4, and also highly dependent on their settings. Hence, different choices will yield different designs, each optimum for the specific design conditions considered.

In this study, the focus was placed upon the global system performance and hydrodynamic behavior of the FOWT. Neither the structural design strength, nor the loading on the structure - implying frequency response analyses -, nor the overall system stability were assessed and integrated in the optimization approach. However, the current functionality already indirectly 
checks the requirement of having the system natural frequencies far from the wave spectra peak frequencies by minimizing the system response, but can also easily be extended by adding more detailed checks, as well as further criteria and optimization objectives, such as for the structural strength or loads and fatigue. Furthermore, a more sophisticated design optimization, implying the design strength but also, for instance, the mooring system, would as well require the definition of additional design variables, such as the wall thickness, the fairlead and anchor positions, the mooring line length, and its extensional stiffness.

In general, the settings have to be selected carefully to ensure that the direct optimization approach can be successful. When still intending an upscaling to a larger MW-class WT, this has to be taken into account in the definition of the boundaries for the allowable value ranges of the design variables, to allow for obtaining a stable FOWT system in the end. Furthermore, there is large freedom in the selection of design variables, both with respect to the variable itself and the number of variables. The optimization objectives will definitely also influence the final optimum design. The objectives selected in this study can be considered as general parameters for the global performance of a FOWT system; however, for more detailed assessment and design of a FOWT system there are almost no limits to the objectives. But still this has to be treated with caution, as this is always subject to a cost-benefit calculation. This aspect was also the reason for just selecting one design-relevant load case, which is then used during the direct optimization approach, instead of running an entire DLC set in each iteration with all individuals. But still it needs to be proved that the obtained optimum FOWT system design performs as required in various environmental conditions. Finally, the achieved optimum design will also depend on the optimization settings; however, due to the preceding study and comparison, it is expected that the selected optimizer NSGAII is suitable for the presented application. The number of simulations to be run is of course again a trade-off between cost or time and benefit and the user has to define up to which accuracy and convergence tolerance the optimization has to be performed. In the end, the final selection of the optimum design out of the huge number of created and simulated individuals depends again on the point of interest and selected approach. However, as shown in Subsection 4.2.2, the resulting optimum designs differ not significantly if the methods are reasonable and the optimization algorithm has run sufficiently long so that a convergence is clearly visible.

\section{CONCLUSION}

In this paper, a direct optimization approach is presented, by which means a floating support structure for a larger MWclass WT is obtained based on an existing smaller FOWT system design without using the intermediate step of upscaling.
This approach is developed in Python programming language. A Python-Modelica framework allows interfacing with FOWT system models defined in the MoWiT library, execution of simulations in Dymola, and programming, as well as utilization of optimization procedures in Python. The input to this direct optimization algorithm only requires minor initial adaptions of the original FOWT system model, as well as the specification of the design conditions, such as the design variables which are to be modified during the optimization, the optimization objectives which should be focused on, the environmental conditions which are used for the system simulation, as well as the optimization settings. Even if the final result highly depends on the choice for these design conditions, an appropriate FOWT system design, which fulfills the specified requirements and performs well with respect to the defined objectives, can be obtained automatically by means of this direct optimization approach.

\section{ACKNOWLEDGMENTS}

This work was partially supported by grant EP/L016303/1 for Cranfield University, University of Oxford and University of Strathclyde, Centre for Doctoral Training in Renewable Energy Marine Structures - REMS (http://www.rems-cdt.ac.uk/) from the UK Engineering and Physical Sciences Research Council (EPSRC).

\section{REFERENCES}

[1] Leimeister, M., 2016. "Rational Upscaling and Modelling of a Semi-Submersible Floating Offshore Wind Turbine". Master Thesis, Delft University of Technology and Norwegian University of Science and Technology and Fraunhofer Institute for Wind Energy Systems.

[2] Jonkman, J., and Musial, W., 2010. Offshore Code Comparison Collaboration (OC3) for IEA Task 23 Offshore Wind Technology and Deployment: Technical Report NREL/TP5000-48191. Technical Report. U.S. Department of Energy.

[3] Popko, W., Thomas, P., Sevinc, A., Rosemeier, M., Bätge, M., Braun, R., Meng, F., Horte, D., Balzani, C., Bleich, O., Daniele, E., Stoevesandt, B., Wentingmann, M., Polman, J. D., Leimeister, M., Schümann, B., and Reuter, A., 2018. IWES Wind Turbine IWT-7.5-164 Rev 4. Fraunhofer Institute for Wind Energy Systems IWES, Bremerhaven.

[4] Jonkman, J., Butterfield, S., Musial, W., and Scott, G., 2009. Definition of a 5-MW Reference Wind Turbine for Offshore System Development: Technical Report NREL/TP-500-38060. Technical Report. U.S. Department of Energy.

[5] Leimeister, M., and Thomas, P., 2017. "The OneWind Modelica Library for Floating Offshore Wind Turbine Simulations with Flexible Structures". In 12th International Modelica Conference, Linköping Electronic Confer- 
ence Proceedings, Linköping University Electronic Press, pp. 633-642.

[6] Thomas, P., Gu, X., Samlaus, R., Hillmann, C., and Wihlfahrt, U., 2014. "The OneWind Modelica Library for Wind Turbine Simulation with Flexible Structure Modal Reduction Method in Modelica". In 10th International Modelica Conference, Linköping Electronic Conference Proceedings, Linköping University Electronic Press, pp. 939-948.

[7] Strobel, M., Vorpahl, F., Hillmann, C., Gu, X., Zuga, A., and Wihlfahrt, U., 2011. "The OnWind Modelica Library for Offshore Wind Turbines - Implementation and first results". In 8th International Modelica Conference, Linköping Electronic Conference Proceedings, Linköping University Electronic Press, pp. 603-609.

[8] Leimeister, M., Kolios, A., Collu, M., and Thomas, P., 2019. "Development and Verification of the OC3 Phase IV Floating Spar-Buoy Wind Turbine System, Utilizing Fraunhofer's MoWiT Library: (to be published)".

[9] Jonkman, J., 2010. Definition of the Floating System for Phase IV of OC3: Technical Report NREL/TP-500-47535. Technical Report. U.S. Department of Energy.

[10] Hansen, M. H., Hansen, A., Larsen, T. J., Øye, S., Sørensen, P., and Fuglsang, P., 2005. Control design for a pitch-regulated, variable speed wind turbine, Vol. 1500 of Ris $\phi$ R, Report. Ris $\varnothing$ National Laboratory, Roskilde.

[11] Leimeister, M., Kolios, A., Collu, M., and Thomas, P., 2019. "Development of a Framework for Wind Turbine Design and Optimization: (to be published)".

[12] Leimeister, M., 2019. "Python-Modelica Framework for Automated Simulation and Optimization". In 13th International Modelica Conference, Linköping Electronic Conference Proceedings, Linköping University Electronic Press, pp. 51-58.

[13] Engineering ToolBox, 2010. Densities of Common Materials: [online] Available at: https://www.engineeringtoolbox.com/density-materialsd_1652.html [Accessed: 12 December 2018].

[14] Engineering ToolBox, 2009.2 Densities of Solids: [online] Available at: https://www.engineeringtoolbox.com/density-solidsd_1265.html [Accessed: 12 December 2018].

[15] Huijs, F., Mikx, J., Savenije, F., and de Ridder, E.-J., 2013. "Integrated design of floater, mooring and control system for a semi-submersible floating wind turbine". In EWEA Offshore, European Wind Energy Association.

[16] Kolios, A., Borg, M., and Hanak, D., 2015. "Reliability analysis of complex limit states of floating wind turbines". JECM, 2(1), pp. 6-9.

[17] Katsouris, G., and Marina, A., 2016. Cost Modelling of Floating Wind Farms: ECN-E-15-078. ECN Report. ECN, Petten, The Netherlands.
[18] Nejad, A. R., Bachynski, E. E., and Moan, T., 2017. “On Tower Top Axial Acceleration and Drivetrain Responses in a Spar-Type Floating Wind Turbine: OMAE2017-62314". In ASME 2017 36th International Conference on Ocean, Offshore and Arctic Engineering, OMAE 2017, The American Society of Mechanical Engineers, p. V009T12A009.

[19] Suzuki, K., Yamaguchi, H., Akase, M., Imakita, A., Ishihara, T., Fukumoto, Y., and Oyama, T., 2011. "Initial Design of Tension Leg Platform for Offshore Wind Farm". JFST, 6(3), pp. 372-381.

[20] International Electrotechnical Commission, 2009. Wind turbines - Part 3: Design requirements for offshore wind turbines: IEC 61400-3, 1 ed. International Standard.

[21] Hadka, D., 2015. Platypus Documentation: Release.

[22] Mytilinou, V., and Kolios, A. J., 2017. "A multi-objective optimisation approach applied to offshore wind farm location selection". Journal of Ocean Engineering and Marine Energy, 3(3), pp. 265-284. 